\title{
ELECTRICAL CHARACTERIZATION OF THIN LAYER MATERIALS: DESTRUCTIVE AND NON-DESTRUCTIVE IRREVERSIBLE PROCESSES IN LANGMUIR FILMS
}

Summary Well-defined Langmuir-Blodgett films prepared by reproducible technique are still highly attractive for exploitation in investigation of subjects as diverse as chain packing, phase transitions, two-dimensional physics, biosensors and membrane physics. During last 15 years the Maxwell displacement current (MDC) measuring technique was developed for dynamic study of monolayers. In experiment MDC flows through the metal electrode/air gap/Langmuir monolayer/water surface structure. Spontaneous formation of the monolayer was observed and significant differences between destructive and non-destructive processes are discussed.

\section{Introduction}

The Langmuir-Blodgett (LB) technique can be exploited to prepare well-ordered monomolecular layer and multilayer films of amphiphilic organic materials. In this technique an insoluble monolayer is formed at the air/water interface by the movable barrier to a specified surface pressure-area state and subsequently transferred onto a solid substrate. Recently with increasing interest in the lowdimensional systems, applications were used in the field of physics, chemistry and biology $[1,2]$. For all these applications it is essential to have a uniform film with well-defined parameters prepared by reproducible technique. Hence, the investigation of irreversible phenomena in the Langmuir film is of prior necessity.

Generally we distinguish between two classes of structural changes induced by mechanical stimulation of the Langmuir film, the transitions between different two-dimensional phases [3], and those in which dimensionality changes from the degree of two to three [4]. The latter class is a destructive process at high pressures caused by the fracture collapse, or at lower pressures (but still above the equilibrium spread pressure [5]) by the slow collapse $[6]$.

In this paper we investigate the formation of a simple fatty acid monolayer at the air-water interface on a pure water subphase and also the significant difference between the non-destructive and destructive (slow collapse) irreversible processes.

\section{Experiment}

The computer-controlled Langmuir trough (Type 611, Nima Technology, England) was used. Stearic acid molecules (Fluka, Switzerland) were spread over the subphase (bidistilled deionized water, $15 \mathrm{M} \Omega . \mathrm{cm}$ ) from a chloroform solution. The Langmuir film was compressed at a rate of $0.002 \mathrm{~nm}^{2} / \mathrm{min}$ per molecule. The
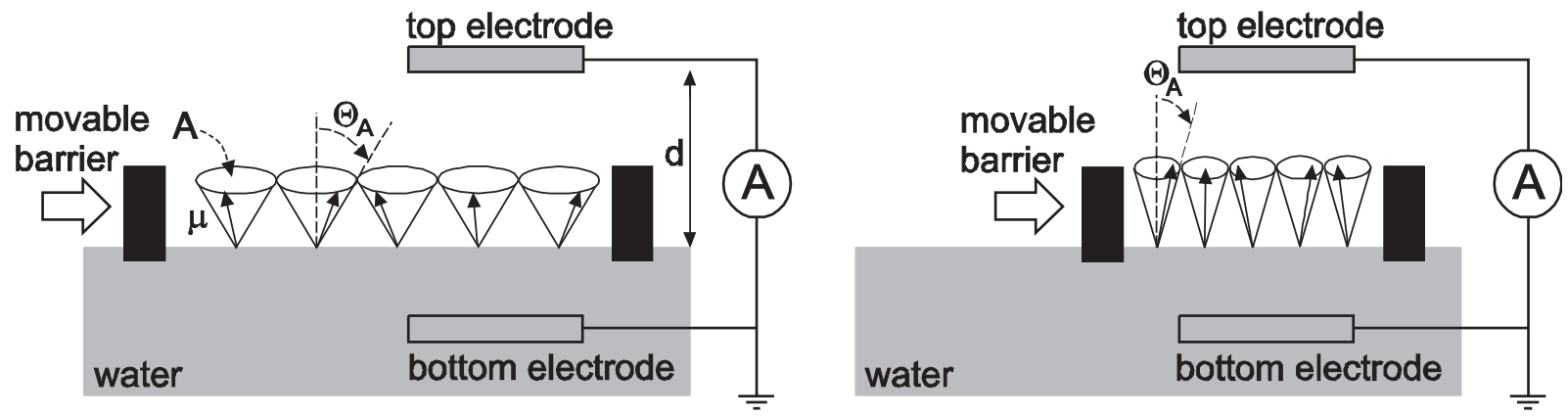

Fig. 1 Sketch of the experimental setup for the displacement current measurement. Rod-like polar molecules execute precessional motion on the water surface with the maximum tilt angle $\Theta_{A}$ (A and $\mu$ stand for the area per molecule and the dipole moment of molecule, respectively), which is controlled by the movable barrier. The electrical shielding of the top electrode is not shown.

\footnotetext{
* Drahoslav Barančok, Július Cirák, Pavol Tomčík, Ján Vajda, Martin Weis

Faculty of Electrical Engineering and Information Technology, Slovak University of Technology

Ilkovičova 3, 81219 Bratislava, Slovak Republic, E-mail: Barancok@elf.stuba.sk
} 
slow collapse experiment was carried out in the isobaric regime, keeping the surface pressure constant at $30 \mathrm{mN} / \mathrm{m}$ for 35 minutes.

Monolayer processes were studied using the Maxwell displacement current (MDC) technique [7,8].

The displacement electric current $I$ flows through a short-circuited capacitor being formed by two parallel plates, the one in air above the monolayer detached at a certain spacing $(1 \mathrm{~mm}$ in our case), the other immersed in the water subphase below the organic monomolecular layer. Compression of the monolayer, carried out by a movable barrier, results in the change of the number of molecules $N$ under the electrode as well as in the mean orientational change of the direction of molecular electric dipoles $\langle\cos \Theta\rangle$. If we consider the organic film as a system of electric dipole moments then it is possible to calculate the induced charge on the upper electrode by the method of images

$$
Q_{i}=\left\langle\mu_{z}\right\rangle N G=\mu\langle\cos \Theta\rangle N G
$$

where $\mu$ is the dipole moment of one molecule ( $\mu_{z}$ is projection of $\mu$ to the normal), $N$ is the number of molecules under the top electrode and $G$ is the geometrical factor of the experimental arrangement. The $\langle\cos \Theta\rangle$ stands for the statistical mean value of $\cos \Theta$, where $\Theta$ is the angle between the dipole moment vector and the normal. Detailed analysis of the dipole moment projection of simple fatty acid was described in [9].

As shown in our previous studies [9], the current flowing in the outer circuit can be expressed as a time change of the induced charge

$$
I=\frac{\partial Q_{i}}{\partial t}=\mu N G \frac{\partial\langle\cos \Theta\rangle}{\partial t}+\mu\langle\cos \Theta \Theta\rangle G \frac{\partial N}{\partial t}(2)
$$

By integrating the displacement current with respect to time, the induced charge $Q$ can be obtained and in this way we also evaluated the vertical component of the molecular dipole moment. Thus, the dipole moment projection to the normal should be calculated as

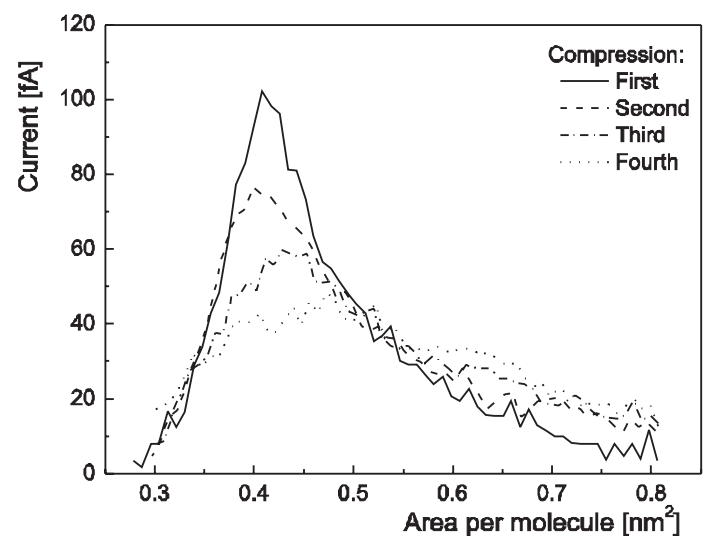

$$
\mu_{z}=\mu\langle\operatorname{coss} \Theta\rangle=\frac{1}{G N} \int I d t
$$

The displacement current technique is sensitive only to dynamic charge processes, which in this arrangement are caused by lateral compression of the monolayer. Therefore any time-independent charge (e.g. polarized water surface or additional substances in subphase) distributed at the interface has no effect. This is advantage in comparison with conventional electrical measurements, e.g. the Kelvin probe method.

\section{Results}

Langmuir film was prepared by standard procedure and was left for $15 \mathrm{~min}$ for solvent evaporation. Measured signals for first four compressions are presented in Fig. 2. On subsequent compressions the signal already remained the same. Although the amplitude of the signal was changed, the area under the current-time isotherm (current-area for constant compression rate) is conserved. The stabilization of the signal (formation of the monolayer) is therefore caused by homogenization of material distribution, not by a destructive process.

The destructive process was modeled by the slow collapse of the Langmuir film. The monolayer collapses were monitored as the relative area loss $\left(A / A_{0}\right)$ with respect to time while keeping the monolayer at a constant surface pressure of $30 \mathrm{mN} / \mathrm{m}$. Recordings of the MDC signals before and after the monolayer degradation are shown in Fig. 3.

Detailed analysis of the change of the dipole moment projection is possible if Eq. 3 is rewritten to the form

$$
\mu_{z}=\frac{1}{G N} \int I d t=\frac{1}{G N} \int \frac{I}{\beta} d A
$$

where $\beta=d A / d t$ is the compression rate per one molecule. Due to the collapse, the number of molecules on the water surface was changed. Hence, at a constant compression rate regime (speed of

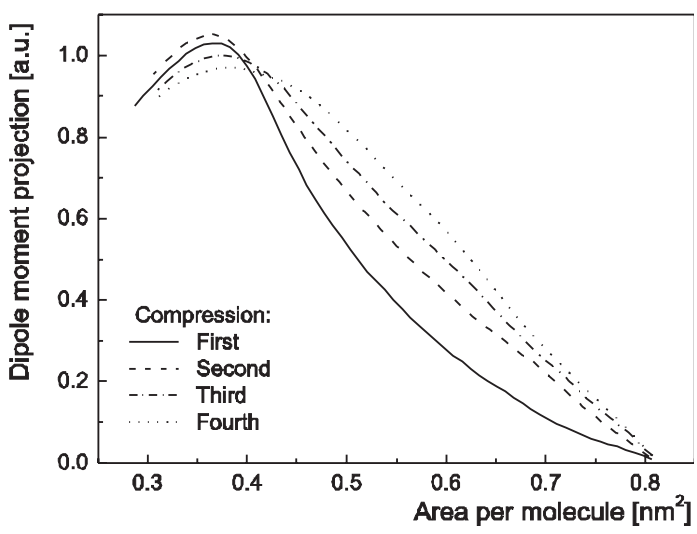

Fig. 2 (top) MDC signals of formation of a stearic acid monolayer during first four compressions. (bottom) Calculations of the dipole moment projection by Eq. 3. 

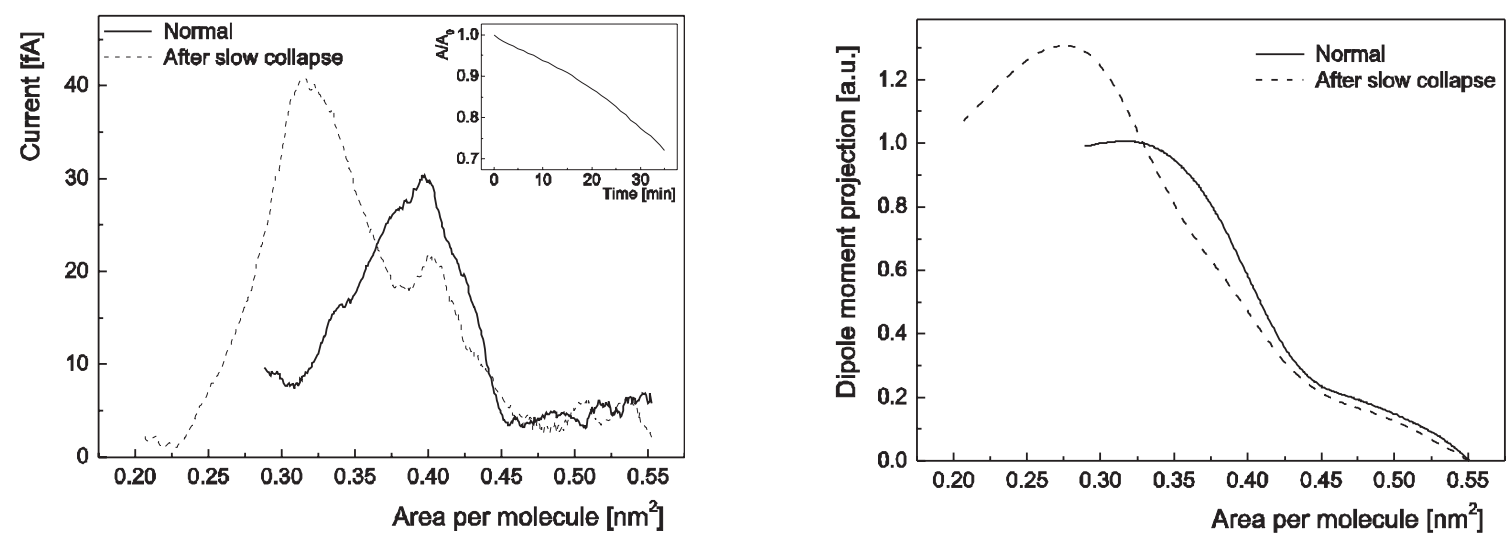

Fig. 3 Left - MDC signals of a stearic acid monolayer before and after the slow collapse. In the insert the relative area loss-time isobar is shown during the slow collapse process.

Right - calculations of the dipole moment projection were accomplished using Eq. 4.

the movable barrier) the compression rate per one molecule was increasing. In our previous work [9] we showed that the ratio $I / \beta$ is conserved. Therefore, the amplitude of the signal as well as the dipole moment (related with the area under current-area isotherm) rise owing to the change of the compression rate $\beta$ and the number of molecules $N$. It is remarkable that the change of the MDC signal is directly proportional to the relative area loss-time isobar.

\section{Conclusions}

For the first time the MDC method was used for studying monolayer collapse. It was shown that its application is highly effective and unambiguously distinguished between destructive and non-destructive processes in the monolayer. The experiment performed on a stearic acid monomolecular film situated on the water surface recognizes homogenization and formation as well as the degree of degradation of the Langmuir film. The destructive process is characterized by the increase of the amplitude and by the shift of the peak towards lower areas. It leads to a significant rise in the calculated dipole moment. The effect of monolayer formation is very important also for the LB technique. It pointed out the need for multiple compressions before LB deposition for building samples with reproducible properties.

\section{Acknowledgement}

The work was supported by the Slovak grant agency VEGA, project No. 1/0277/03 and by Agency for Promotion of Research of Development No. APVT-51-013907.

\section{References}

[1] SACKMANN, E.: Science, 271 (1996), 43.

[2] LIPOWSKY, R.: Nature, 349 (1991), 475.

[3] VOLLHARDT, D., FAINERMAN, V.: Colloids Surf. A 176 (2001) 117.

[4] VOLLHARDT, D.: Colloids Surf. A 143 (1998) 185.

[5] GAINES, G. L.: Insoluble Monolayers at Liquid/Gas Interfaces (Wiley-Interscience, New York, 1966).

[6] SMITH, R. D., BERG, J. C.: Colloid Interface. Sci. 74 (1980) 273.

[7] MAJIMA, Y., IWAMOTO, M.: Rev. Sci. Instrum. 62(9) (1991) 2228.

[8] BARANČOK, D., CIRÁK, J., TOMČÍK, P., VAJDA, J.: Phys. Stat. Sol. 169(2) (1998) 267.

[9] VAJDA, J, WEIS, M., BARANČOK, D., CIRÁK, J., TOMČÍK, P.: App. Surf. Sci. 229/1-4 (2004) 183. 